\title{
Leptin-mediated regulation of ICAM-1 is Rho/ROCK dependent and enhances gastric cancer cell migration
}

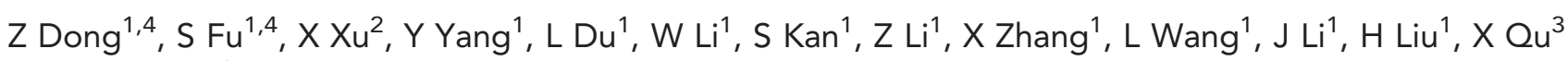 \\ and C Wang ${ }^{*}, 1$
}

${ }^{1}$ Department of Clinical Laboratory, Qilu Hospital, Shandong University, 107 Wenhuaxi Road, Jinan 250012, Shandong, China; 2 Department of Obstetrics and Gynecology, Qilu Hospital, Shandong University, 107 Wenhuaxi Road, Jinan 250012, Shandong, China and ${ }^{3}$ Institute of Basic Medical Sciences, Qilu Hospital, Shandong University, 107 Wenhuaxi Road, Jinan 250012, Shandong, China

Background: Our previous study indicates that leptin enhances gastric cancer (GC) invasion. However, the exact effect of leptin on GC metastasis and its underlying mechanism remain unclear. Intercellular adhesion molecule-1 (ICAM-1), a major molecule in stabilising cell-cell and cell-extracellular matrix interactions, is overexpressed and has crucial roles in tumour metastasis.

Methods: Here, we investigated leptin and ICAM-1 expression in GC tissues. Furthermore, we characterised the influence of leptin on ICAM-1 expression in GC cells and elucidated the underlying mechanism.

Results: Leptin and ICAM-1 were overexpressed in GC tissues, and a strong positive correlation was observed. They were also related with clinical stage or lymph node metastasis. Furthermore, leptin induced GC cell (AGS and MKN-45) migration by upregulating ICAM-1, and knockdown of ICAM-1 by small interference RNA (siRNA) blocked this process. Cell surface ICAM-1, as well as soluble ICAM-1 (sICAM-1), was also enhanced by leptin. Moreover, leptin increased ICAM-1 expression through Rho/ROCK pathway, which was attenuated by pharmacological inhibition of Rho (C3 transferase) or its downstream effector kinase Rho-associated protein kinase (ROCK) (Y-27632).

Conclusions: Our findings indicate that leptin enhances GC cell migration by increasing ICAM-1 through Rho/ROCK pathway, which might provide new insight into the significance of leptin in GC.

Gastric cancer (GC) ranks as the second leading cause of cancerrelated death in the world (Parkin et al, 2005). The high aggressiveness and rapid metastasis of GC contribute to its high mortality rate (Wang and Chen, 2011). The metastasis involves multiple factors, such as obesity, an important risk factor in GC (Calle et al, 2003; Howard et al, 2010). Adipocytes provide fatty acids for rapid tumour growth, and the dysfunction of lipid metabolism can lead to the pathogenesis of human GC (Song et al, 2012). Leptin is an adipokine of the obesity ( $o b)$ gene, and has been known to have roles in the homeostasis of body weight by regulating food intake and energy metabolism (Chan et al, 2006). Recent studies have revealed that leptin is implicated in the tumorigenesis of cancer, such as ovarian cancer, endometrial cancer, GC, and so on (Zhao et al, 2007; Wu et al, 2012; Xu et al, 2013). Leptin exerts its function through leptin receptor, and the use of leptin receptor antagonists has been recognised as a potential therapeutic approach for cancer (Otvos et al, 2011). Our previous study indicates that leptin enhances GC cell invasion by upregulating the expression of membrane type 1-matrix metalloproteinase (MT1-MMP) (Dong et al, 2013). Converging studies

*Correspondence: Professor C Wang; E-mail: cxwang@sdu.edu.cn
${ }^{4}$ These authors contributed equally to this work.

Received 27 September 2013; revised 19 December 2013; accepted 21 January 2014; published online 18 February 2014

(c) 2014 Cancer Research UK. All rights reserved 0007 - 0920/14 
reveal that leptin expression is elevated in GC patients, and is also significantly correlated with GC metastasis (Shida et al, 2005; Ishikawa et al, 2006; Zhao et al, 2007). However, the exact effect of leptin on GC metastasis and its underlying mechanism remain poorly understood.

Recent findings of upregulation of intercellular adhesion molecule-1 (ICAM-1, also known as CD54) by leptin in allergic inflammation may shed light on how leptin modulates cell migration (Wong et al, 2007). This suggests a link between leptin and ICAM-1. ICAM-1 is a transmembrane glycoprotein of the immunoglobulin superfamily. It binds to two major ligands, lymphocyte function-associated antigen-1 (LFA-1, CD11a/CD18) and macrophage- 1 antigen (Mac-1, CD11b/CD18). LFA-1 and Mac-1 expressed on NK cell and macrophages share a common $\beta$ chain (Diamond et al, 1990; Zimmerman and Blanco, 2008). ICAM-1 has an essential role in cell-cell and cell-extracellular matrix interaction. Binding to LFA-1 and Mac-1, ICAM-1 can facilitate the metastasis of cancer cells by escaping the recognition and attack of immunocytes (Makrilia et al, 2009). Previous studies show that ICAM-1 is associated with more aggressive lesions in prostate cancer (Chen et al, 2012), lung cancer (Grothey et al, 1998), and GC (Maruo et al, 2002). Laboratory models have demonstrated that the upregulation of ICAM-1 can promote tumour cell invasion and metastasis. In addition, overexpressed ICAM-1 may be released from the local cancer cell nests and enter the serum as sICAM-1 (Essick et al, 2008). Elevated serum ICAM-1 level is correlated significantly with serum CA19-9 and CEA in GC (Polychronidis et al, 2003); consistently, suppression of ICAM-1 expression is related to attenuated tumour progression and metastasis (Kaliora et al, 2008; Chen et al, 2012; Yu et al, 2012). Interestingly, both leptin and ICAM-1 are increased after Helicobacter pylori infection (Fan et al, 1995; Azuma et al, 2001). To our knowledge, there is no published data about the effect of leptin on ICAM-1 and its role in GC metastasis.

The expression of ICAM-1 may be regulated by a variety of signalling pathways, such as Rho (the small $G$ protein Ras homologue gene family) and its immediate downstream effector Rho-associated coiled-coil-forming protein kinase (ROCK) (Jung et $a l, 2012)$. ROCK is ubiquitously expressed serine-threonine protein kinases that are involved in diverse cellular functions, including actin cytoskeleton organisation, cell adhesion, and motility (Burridge and Wennerberg, 2004). Hyperactivation of the Rho/ROCK pathway is known to be linked with more aggressive tumour properties such as metastasis (Sahai and Marshall, 2002). Rho/ROCK may be regulated by some factors in tumours. For example, lysophosphatidic acid triggers focal adhesion assembly through Rho/ROCK pathway in human ovarian cancer cells (Sawada et al, 2002). A previous study reveals that leptin may induce Rho/ROCK pathway (Schram et al, 2011). On the basis of these reports, we hypothesise that Rho/ROCK pathway may be involved in the leptin-mediated expression of ICAM-1. This study has demonstrated that leptin promotes the migration of GC cells by upregulating ICAM-1 expression via the involvement of Rho/ROCK pathway. Targeting ICAM-1 and its regulation pathway might provide preliminary experimental clues for the development of therapies against the metastasis of GC.

\section{MATERIALS AND METHODS}

Patients and specimens. Archived paraffin-embedded GC tissues and matched adjacent normal gastric tissues were collected from 84 patients who underwent surgery for primary gastric carcinoma in the Department of General Surgery, Qilu Hospital, Shandong University (Shandong, China). The clinical parameters and pathologic classifications are presented and summarised in
Table 1. There were 34 males (age, 31-77 years; median, 57 years) and 50 females (age, 32-75 years; median, 53 years); all patients had not received medical therapy before surgery. The diagnosis of each case was confirmed by two pathologists. The research was approved by the Ethics Committee of Qilu Hospital and we obtained written informed consent from all patients.

Reagents and Antibodies. Rabbit anti-human ICAM-1 polyclonal antibody for immunohistochemistry and western blot (WB) and anti- $\beta$-actin $\mathrm{mAb}$ were obtained from Cell Signaling Technology (Boston, MA, USA). Rabbit anti-human leptin polyclonal antibody was from Santa Cruz Biotechnology, Inc. (Santa Cruz, CA, USA). Recombinant human leptin (rhleptin), mouse anti-human ICAM-1 $\mathrm{mAb}$ for flow cytometry, sICAM-1 commercial ELISA (enzymelinked immunosorbent assay) kit were obtained from $R \& D$ Systems (Minneapolis, MN, USA). Rabbit anti-human phosphoROCK polyclonal antibody was purchased from AnaSpec Inc. (Fremont, CA, USA). Rat anti-human ROCK was obtained from BD Biosciences (San Jose, CA, USA). PE-goat anti-mouse secondary mAb was purchased from Invitrogen (Carlsbad, CA, USA). All isotype controls were purchased from corresponding vendors.

GC cell lines and cell culture. The GC cell lines AGS and MKN45 from the Culture Collection of the Chinese Academy of Sciences (Shanghai, China) and the Riken Cell Bank (Tsukuba, Japan) were grown in DMEM/F12 medium containing 10\% fetal calf serum (FCS) (Hyclone-Pierce, Rockford, IL, USA). Cells were seeded upon $80 \%$ confluence. In the following experiments, cells were serum-deprived for $12 \mathrm{~h}$ prior to the administration of exogenous stimulation, and they were then incubated with serum-free DMEM/F12 medium except for special indications.

Immunohistochemical analysis. Paraffin-embedded tissues were deparaffinised and rehydrated. After antigen retrieval, all tissues were exposed to respective primary antibodies (leptin, 1:400; ICAM-1, $1: 1000$ ) overnight at $4{ }^{\circ} \mathrm{C}$, and then incubated with biotin-conjugated secondary antibody for $30 \mathrm{~min}$ at $37^{\circ} \mathrm{C}$, followed by peroxidase-conjugated avidin. Staining was observed with $\mathrm{DAB}$ (3,3N-diaminobenzidine tertrahydrochloride), and evaluated by two pathologists. An immunohistochemical score was generated by incorporating both the percentage of positive tumour cells and the intensity of staining as previously described (Yang et al, 2011).

Transient transfection in AGS cells. The vector pcDNA 3.1/mycHis A was kindly provided by Dr Yinghong Xiao (Raul Andino lab, Department of Microbiology and Immunology, University of California, San Francisco, CA, USA). The human full-length ICAM-1 cDNA (Genebank: NM_000201)) was amplified and subcloned between the EcoR I and BamH I sites of the vector to construct eukaryotic expression vector pcDNA3.1-ICAM-1 in Central Research Laboratory (Bethune Second Hospital of Jilin University, Changchun, China). About $2 \times 10^{5}$ cells per well were seeded onto six-well plates, and then transfected with pcDNA3.1ICAM-1 or an empty vector (MOCK) as control, using Lipofectamine 2000 following the manufacturer's protocol. After $24 \mathrm{~h}$, the transfection efficacy was evaluated.

Cell migration assay. The migration assay was performed using transwell inserts ( $8 \mu \mathrm{m}$ pores, BD Biosciences, Bedford, MA, USA) in 24-well dishes as previously described (Chen et al, 2012). Two hundred microliters of serum-free medium containing $1 \times 10^{4}$ cells were seeded in the upper chamber. The lower chamber was filled with $600 \mu \mathrm{l}$ medium containing 10\% FCS. Cells were incubated for $1 \mathrm{~h}$ for adherence, and leptin was then added in the upper chamber for another $24 \mathrm{~h}$ at $37^{\circ} \mathrm{C}$ in $5 \% \mathrm{CO}_{2}$. After $24 \mathrm{~h}$, the non-migrated cells were gently scraped off by cotton swab. The migrated cells were fixed by $10 \%$ formalin for $5 \mathrm{~min}$, stained with eosin, and counted. Three fields of each well were photographed, and the cell 
Table 1. Relationship between leptin, ICAM-1 expression, and clinicopathological variables.

\begin{tabular}{|c|c|c|c|c|c|c|c|}
\hline & & \multicolumn{3}{|c|}{ Leptin } & \multicolumn{3}{|c|}{ ICAM-1 } \\
\hline Parameters & No. of patients & $-1+$ & $++/+++$ & $P$-value & $-1+$ & $++/+++$ & $P$-value \\
\hline \multicolumn{8}{|l|}{ Gender } \\
\hline Male & 34 & 13 & 21 & 0.541 & 12 & 22 & 0.764 \\
\hline Female & 50 & 23 & 27 & & 18 & 32 & \\
\hline \multicolumn{8}{|l|}{ Age } \\
\hline$>50$ years & 52 & 24 & 28 & 0.135 & 22 & 30 & 0.687 \\
\hline$\leqslant 50$ years & 32 & 12 & 20 & & 8 & 24 & \\
\hline \multicolumn{8}{|l|}{ BMI } \\
\hline & 84 & 36 & 48 & 0.541 & 30 & 54 & 0.554 \\
\hline \multicolumn{8}{|l|}{ Tumour size } \\
\hline$\leqslant 5 \mathrm{~cm}$ & 41 & 17 & 24 & 0.212 & 16 & 25 & 0.147 \\
\hline$>5 \mathrm{~cm}$ & 43 & 19 & 24 & & 14 & 29 & \\
\hline \multicolumn{8}{|c|}{ Differentiation } \\
\hline Well & 25 & 5 & 20 & 0.395 & 8 & 17 & 0.786 \\
\hline Moderate & 28 & 18 & 10 & & 11 & 17 & \\
\hline Poor & 31 & 13 & 18 & & 11 & 20 & \\
\hline \multicolumn{8}{|c|}{ Borrmann-type } \\
\hline I & 21 & 12 & 9 & 0.143 & 8 & 13 & 0.278 \\
\hline$\|$ & 19 & 9 & 10 & & 4 & 15 & \\
\hline III & 28 & 7 & 21 & & 13 & 15 & \\
\hline IV & 16 & 8 & 8 & & 5 & 11 & \\
\hline \multicolumn{8}{|c|}{ Invasion depth } \\
\hline T1 & 9 & 2 & 7 & 0.589 & 3 & 6 & 0.232 \\
\hline T2 & 15 & 5 & 10 & & 8 & 7 & \\
\hline T3 & 26 & 16 & 10 & & 10 & 16 & \\
\hline T4 & 34 & 13 & 21 & & 9 & 25 & \\
\hline \multicolumn{8}{|c|}{ Lymph nodes metastasis } \\
\hline NO & 11 & 4 & 7 & $0.021^{*}$ & 7 & 4 & $0.001^{\star *}$ \\
\hline N1 & 21 & 10 & 11 & & 13 & 8 & \\
\hline N2 & 23 & 11 & 12 & & 7 & 16 & \\
\hline N3 & 29 & 11 & 18 & & 3 & 26 & \\
\hline \multicolumn{8}{|c|}{ Invaded adjacent organs } \\
\hline Yes & 26 & 11 & 15 & 0.243 & 8 & 18 & 0.089 \\
\hline No & 58 & 25 & 33 & & 22 & 36 & \\
\hline \multicolumn{8}{|c|}{ Clinical stage } \\
\hline I & 14 & 9 & 5 & $0.011^{* *}$ & 7 & 7 & $0.028^{*}$ \\
\hline II & 16 & 8 & 8 & & 5 & 11 & \\
\hline III & 21 & 11 & 10 & & 8 & 13 & \\
\hline IV & 33 & 8 & 25 & & 10 & 23 & \\
\hline
\end{tabular}

numbers were determined by Kodak MI software. Each experiment was performed in double and repeated three times. The number of migrating cells in each experiment was corrected for proliferation effects using a cell viability assay (corrected migrating cell number $=$ counted migrating cell number/percentage of viable cells).

RT-PCR and real-time RT-PCR. Total RNA was extracted from human GC cells using TRIzol reagent (Invitrogen), and $1 \mu \mathrm{g}$ of total RNA was reverse transcribed using M-MuLV reverse transcriptase (Fermentas, St Leon-Rot, Germany) for $60 \mathrm{~min}$ at $42{ }^{\circ} \mathrm{C}$ in the presence of the oligo-dT primer. Reverse transcription-polymerase chain reaction (RT-PCR) was performed as previously described (Xu et al, 2012). The products were separated on $1.8 \%$ agarose gel and visualised by ethidium bromide. For real-time RT-PCR, cDNA was amplified in triplicate with the LightCycler FastStart DNA Master SYBR Green I Reagent 
Kit (Roche Molecular Biochemicals, Mannheim, Germany) in the LightCycler 2.0 (Roche Applied Science, Mannheim, Germany) according to the manufacturer's instruction. The amplification condition was at $95^{\circ} \mathrm{C}$ for $15 \mathrm{~min}$, followed by 40 cycles $(15 \mathrm{~s}$ at $95^{\circ} \mathrm{C} ; 1 \mathrm{~min}$ at $60^{\circ} \mathrm{C}$ ). Melting curve analysis was used to confirm amplification specificity. The quantification data were analysed with LightCycler analysis software version 4.0 (Roche Applied Science). The average $C_{t}$ of triplicate wells was calculated as the amount of gene product present in the sample. The relative gene expression level was determined by the ratio between $C_{\mathrm{t}}$ value for ICAM- 1 and $\beta$-actin. The primer sequences used in this study were as follows: ICAM-1 primer for real-time RT-PCR: sense 5'-GCCACTTCTTCTGTAAGTCTGTGGG-3', anti-sense 5'-CTAC CGGCCCTGGGACG-3'. ICAM-1 primer for RT-PCR: sense $5^{\prime}$-CTGCTGGGAATTTTCTGGCCAA- ${ }^{\prime}$, anti-sense $5^{\prime}$-CTATGG CAACGACTCCTTCTCG- $3^{\prime}$. $\beta$-actin: sense $5^{\prime}$-AGCGAGC ATCCCCCAAAGTT-3', anti-sense $5^{\prime}$-GGGCACGAAGGCTCA TCATT- $3^{\prime}$.

WB analysis. The cultured cells were lysed on ice as described previously (Xu et al, 2012). Equal amounts of proteins from each sample were separated on $10 \%$ SDS-PAGE gels and transferred onto nitrocellulose membranes. Membranes were incubated with respective primary antibodies (ICAM-1 $(1: 500)$, ROCK $(1: 1000)$, P-ROCK $(1: 1000)$, and $\beta$-actin $(1: 2000))$ overnight at $4{ }^{\circ} \mathrm{C}$. The specific horseradish peroxidase-conjugated goat anti-rabbit or goat anti-rat secondary antibody was used to blot the target proteins, and the immunoreactivity of the target proteins was assessed using an enhanced chemiluminescence detection system.

Flow cytometric analysis. Cells were collected, washed three times with PBS containing $0.1 \%$ BSA. Cells were incubated with antiICAM-1 mAb for $30 \mathrm{~min}$ at room temperature and then incubated with PE-goat anti-mouse secondary $\mathrm{mAb}$ for $30 \mathrm{~min}$. After another three washes, cells were suspended in PBS containing 0.1\% BSA and then immediately analysed by FACSCalibur (BD Biosciences). Data were analysed by the software WinMDI 2.8 .

Enzyme-linked immunosorbent assay. GC cells (AGS, MKN-45) $\left(2 \times 10^{5}\right.$ cells per well $)$ were seeded onto six-well plate. Cells were serum-deprived for $12 \mathrm{~h}$ when confluence attained $80 \%$. Then the medium was removed, and cells were treated in the absence (control) or presence of leptin $\left(100 \mathrm{ng} \mathrm{ml}^{-1}\right)$ after $1 \mathrm{~h}$ pretreatment with C3 transferase $\left(0.25 \mu \mathrm{g} \mathrm{ml}^{-1}\right)$ or Y-27632 $(3.3 \mu \mathrm{M})$. The inhibitors were present for the duration of leptin treatment. After $24 \mathrm{~h}$ at $37^{\circ} \mathrm{C}$ in $5 \% \mathrm{CO}_{2}$, cell culture supernatants were collected and centrifuged at $300 \times \mathrm{g}$ for $5 \mathrm{~min}$. The levels of sICAM-1 in the cell-free supernatants were detected using a commercial ELISA kit according to the manufacturer's instruction.

RNA interference. Small interference RNA constructs targeting ICAM-1 and stable negative control (NC) were designed and purchased from GenePharma Co., Ltd (Shanghai, China). For transient silencing, $2 \times 10^{5} \mathrm{ml}^{-1}$ cells were seeded onto six-well plates, and transfected with ICAM-1-siRNA $\left(80 \mathrm{nmoll}^{-1}\right)$ using Lipofectamine RNAiMAX reagent (Invitrogen), following the manufacturer's protocol. The interference efficiency was evaluated via $\mathrm{WB}$ analysis. The sequence for ICAM-1-siRNA was: sense strand: $5^{\prime}$-GCCUCAGCACGUACCUCUATT-3', antisense strand: $5^{\prime}$-UAGAGGUACG UGCUGAGGCTT- $3^{\prime}$. The sequence for control siRNA was: sense strand: 5'-UUCUCCGAACGUGUCACGUTT- ${ }^{\prime}$, antisense strand: $5^{\prime}$-ACGUGACACGUUCGGAG AATT-3'.

RhoA GTPase activity assay. RhoA GTPase activity was detected using the G-LISA RhoA activation assay kit (Cytoskeleton Inc., Denver, CO, USA) according to the manufacturer's instructions. Briefly, cells were lysed, and equal amount of protein from each sample was added to precoated Rho-GTP binding plates. After $30 \mathrm{~min}$ of shaking at $4{ }^{\circ} \mathrm{C}, 200 \mu \mathrm{l}$ of antigen-presenting buffer were added to each well and incubated for $2 \mathrm{~min}$ at room temperature. Following the conjugation of the anti-actin and secondary antibodies, RhoA activity was quantified using the absorbance at $490 \mathrm{~nm}$.

Statistical analysis. Data were primarily presented as the mean \pm s.d. The SPSS software package (version 13.0; SPSS, Chicago, IL, USA) was used for all statistical analysis. The distribution of the samples was determined via KolmogorovSmirnov test. Correlations of leptin and ICAM-1 expression with clinicopathological factors were analysed by Kruskal-Wallis test or Mann-Whitney U-test, as appropriate. Chi-squared test was applied to analyse the correlation of leptin and ICAM-1, respectively. Other data from experiments were analysed by paired Student's $t$-test or one-way ANOVA wherever appropriate. Tukey post hoc comparison or SNK test was performed when statistical significance $(P<0.05)$ was found between observations. Each variable was tested twice, and the experiment was repeated three times.

\section{RESULTS}

Expression of leptin and ICAM-1 in primary GC lesions was related with tumour progression. To investigate the correlation of leptin and ICAM-1 in GC and whether these proteins were involved in GC progression, the expression of leptin and ICAM-1 was immunohistochemically detected in 84 carcinoma tissues and matched with adjacent normal tissues. Moderate to strong $(++1$ $+++)$ immnoreactivity for leptin and ICAM-1 was identified in $57.1 \%(48 / 84)$ and $64.2 \%(54 / 84)$ of carcinomas, respectively. In contrast, only moderate immunoreactivity $(++)$ for these markers were detected in $44.3 \%$ and $17.8 \%$ of normal tissues, respectively (Figure $1 \mathrm{~A}$ and $\mathrm{B}$, Table 1 ). Immunostains revealed that leptin and ICAM-1 were expressed both in the cytoplasm and on the cell surface, but not in the nucleus (Figure 1A). Moreover, leptin and ICAM-1 were positively correlated with lymph nodes metastasis and clinical stage (Table 1), and the levels of both proteins were significantly correlated with each other (Figure 1C).

Leptin induced the migration of human GC cells. Our precious study showed that leptin could promote GC cell invasion (Dong et al, 2013). Some studies indicated that leptin induced migration of several tumours such as endometrial cancer and glioma (Yeh et al, 2009). To investigate the effects of leptin on GC cell migration, we first examined the levels of leptin and leptin receptor in two GC cell lines (AGS and MKN-45). RT-PCR and WB analysis revealed that leptin and all leptin receptors were expressed in both cell lines, though the level of leptin was far lower than the physiological level (Dong et al, 2013). Our data showed that leptin (10-500 $\left.\mathrm{ng} \mathrm{ml}^{-1}\right)$ treatment significantly enhanced cell migration, with a maximal response at the dose of $500 \mathrm{ng} \mathrm{ml}^{-1}(P<0.001$ for both cell lines) compared with controls (Figure $2 \mathrm{~A}$ and $\mathrm{B}$ ). Moreover, a maximal response to leptin $\left(100 \mathrm{ng} \mathrm{ml}^{-1}\right)$ was observed at $36 \mathrm{~h}$ (Figure 2C).

ICAM-1 was involved in leptin-induced GC cell migration. Previous studies reveal that ICAM-1 has an essential role in cancer cell migration (Maruo et al, 2002). To investigate whether ICAM-1 is regulated by leptin $\left(100 \mathrm{ng} \mathrm{ml}^{-1}\right.$, a dose that could induce a significant effect), we examined ICAM-1 expression after being treated with leptin. The results of RT-PCR demonstrated that leptin significantly increased ICAM-1 expression in AGS and MKN-45 cells (Figure 3A; $4.06 \pm 0.54$-fold for AGS, $P<0.001$; $2.56 \pm 0.33$-fold for MKN-45, $P=0.005)$. The similar trend was also observed in the results of real-time RT-PCR (Figure $3 \mathrm{~B}$ ) and the protein level (Figure 3C; $3.07 \pm 0.25$-fold for AGS, $P<0.001$; $2.9 \pm 0.26$-fold for MKN-45, $P=0.003$ ). As shown in Figure 3D, 

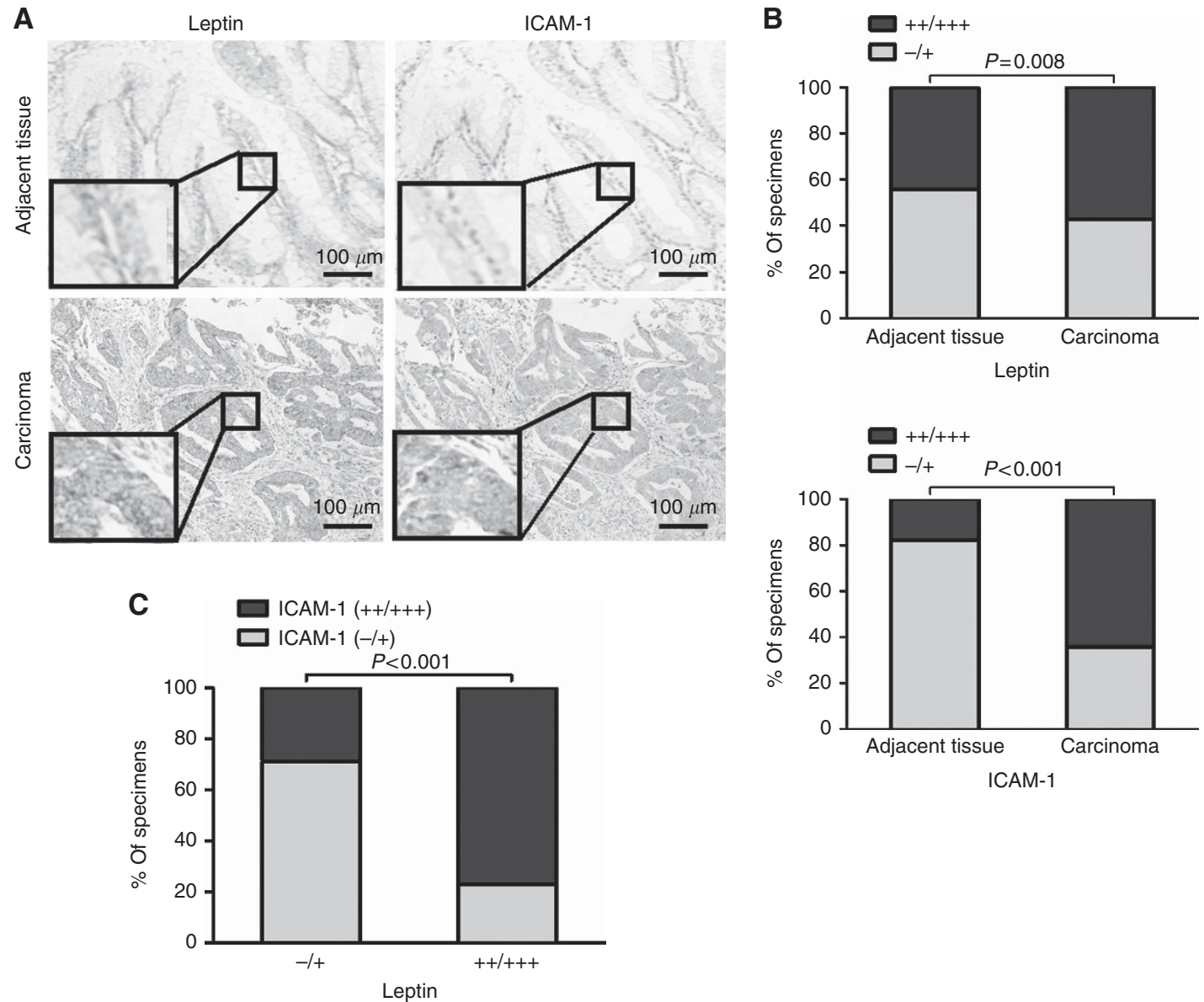

ICAM-1

Figure 1. Expression levels of leptin and ICAM-1 in human gastric carcinoma and adjacent tissues. (A) Immunohistochemical staining of leptin and ICAM-1 in human gastric carcinoma and adjacent tissues. Two representative specimens are shown. Scale bar, $100 \mu \mathrm{m}$. (B) The corresponding percentages of specimens with low $(-/+)$ or high $(++/+++)$ were summarised. (C) Percentages of specimens with low $(-/+)$ or high $(++/+++)$ leptin expression in which ICAM-1 low $(-/+)$ or high $(++/+++)$ observed.

the cell surface ICAM-1 was also induced by leptin $\left(100 \mathrm{ng} \mathrm{ml}^{-1}\right)$, as evidenced by the increased percentage of positive cells (AGS: from $43.65 \% \pm 2.42 \%$ to $78.96 \% \pm 2.09 \%, P<0.001$; MKN-45: from $35.35 \% \pm 1.61 \%$ to $59.64 \% \pm 3.51 \%, \quad P<0.01)$. Furthermore, sICAM-1 level in the supernatant of cultured cells (AGS and MKN-45) was also elevated after leptin treatment $(P<0.05$, Figure $3 \mathrm{E})$.

To test whether leptin-mediated ICAM-1 upregulation was involved in GC cell migration, we first transduced GC cells to overexpress ICAM-1 (pcDNA3.1-ICAM-1). The transfection efficacy was analysed by WB (Figure 4A). Our findings showed that overexpressed ICAM-1 could promote GC cell migration (Figure 4B). Moreover, we performed knockdown of ICAM-1 by siRNA. The knockdown efficiency was evaluated by WB (Figure 4C). As shown in Figure 4D, leptin-induced migration of GC cells was significantly reduced by transient transfection of ICAM-1-siRNA (AGS: $53.9 \% \pm 3.6 \%, \quad P=0.020 ; \quad$ MKN-45: $42.79 \% \pm 3.78 \%, P=0.005)$. The NC group was transfected with control siRNA.

Leptin increased the expression of ICAM-1 through Rho/ROCK pathway. Rho GTPases (e.g., RhoA, Racl, and Cdc42) is the binary switch between an active GTP-bound form and an inactive GDP-bound form. A previous study described that Rho/ROCK signalling was related with GC (Matsuoka et al, 2011). Therefore, we investigated whether leptin modulated RhoA activity in GC cells. As shown in Figure 5A, the RhoA activity was significantly increased after leptin stimulation at $15 \mathrm{~min}$ (1.2 \pm 0.2 -fold for AGS; $1.1 \pm 0.1$-fold for $\mathrm{MKN}-45), 30 \mathrm{~min}$ (1.6 \pm 0.1 -fold for AGS;
$1.36 \pm 0.06$-fold for $\mathrm{MKN}-45$ ), and $60 \mathrm{~min}$ (1.67 \pm 0.12 -fold for AGS; $1.5 \pm 0.1$-fold for MKN-45). Furthermore, the phosphorylation of ROCK was also induced by leptin in AGS and MKN-45 cells at 15, 30, and $60 \mathrm{~min}$, though the total ROCK expression remained unaltered over the time course of leptin treatment in these studies (Figure 5B).

To further explore whether leptin regulated the expression of ICAM-1 through the Rho/ROCK pathway, cells were pretreated with the specific pharmacological inhibitor C3 transferase (Rho inhibitor; Cytoskeleton) at $0.25 \mu \mathrm{g} \mathrm{ml}^{-1}$ or Y-27632 (ROCK inhibitor; Calbiochem, Nottingham, UK) at $3.3 \mu \mathrm{M}$ (Schram et al, 2011), followed by leptin treatment. The expression of ICAM-1 was analysed by WB, flow cytometry, and ELISA (for sICAM-1). As shown in Figure 5C, pretreatment with either Rho (C3 transferase) or ROCK (Y-27632) inhibitor prevented the leptin-induced upregulation of ICAM-1 in AGS and MKN-45 cells, and there was no difference between C3 transferase and Y-27632. Although treatment with inhibitors alone resulted in a subtle decrease in the expression of ICAM-1 compared with control, the inhibitors alone did not influence the expression of ICAM-1. A similar trend was also observed with the cell surface ICAM-1 (Figure 5D) and sICAM-1 (Figure 5E).

\section{DISCUSSION}

Metastasis, such as lymph node metastasis, is a common cause of death from GC (Wang and Chen, 2011). Leptin has been 
A Leptin $\left(\mathrm{ng} \mathrm{ml}^{-1}\right)$
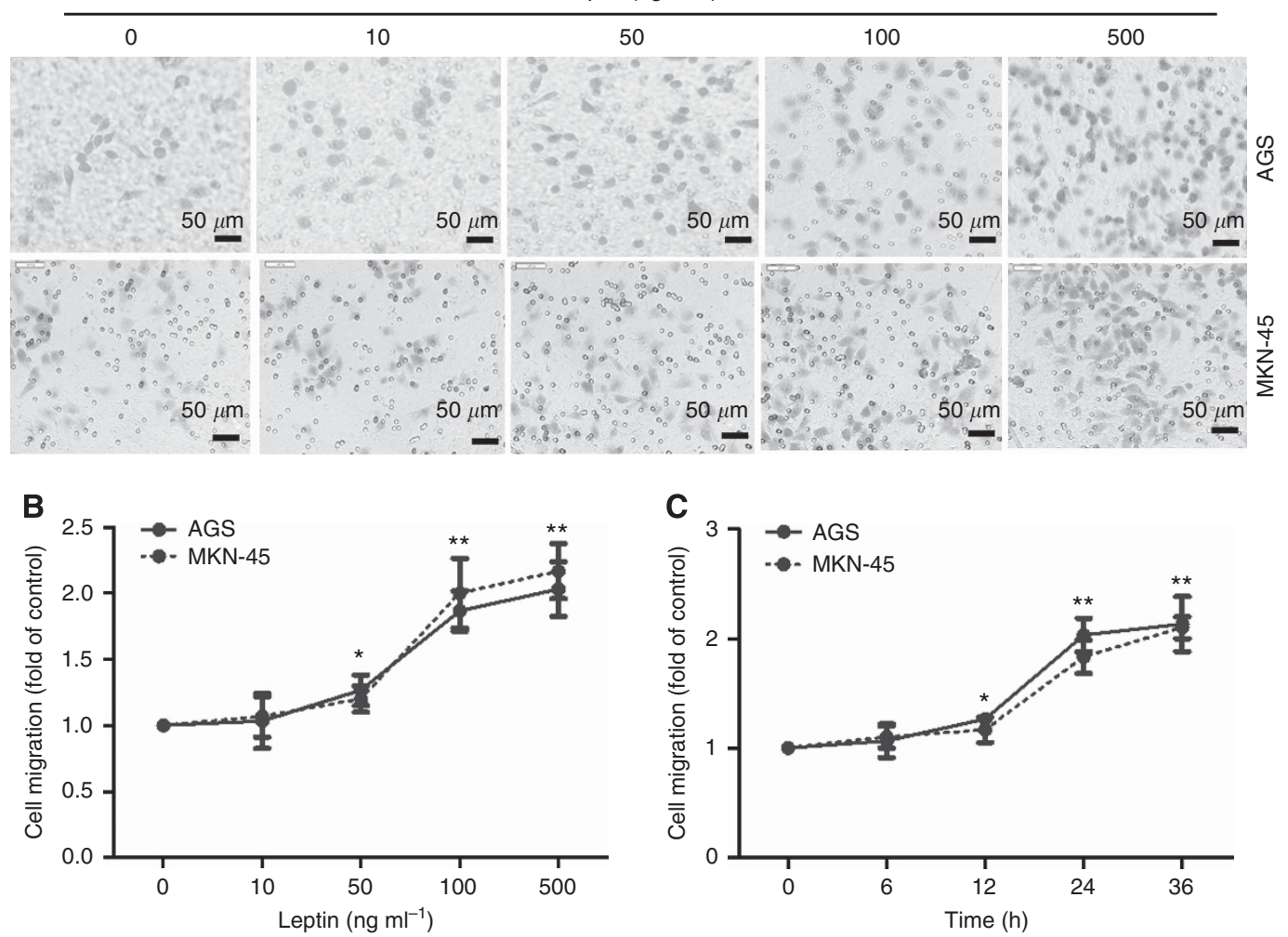

Figure 2. Effect of leptin on the migration of GC cells. (A) AGS and MKN-45 cells $\left(1 \times 10^{4}\right.$ cells/well) were treated in the absence (control) or presence of various doses of leptin $\left(10,50,100\right.$, and $500 \mathrm{ng} \mathrm{ml}^{-1}$ ) for $24 \mathrm{~h}$. The migration was measured using transwell assay. Scale bar, $50 \mu \mathrm{m}$. The migration numbers were expressed relatively to control (B). (C) In a separate experiment, AGS and MKN-45 cells were treated with leptin $\left(100 \mathrm{ng} \mathrm{ml}^{-1}\right)$, and the migration was detected at $0,6,12,24$, and $36 \mathrm{~h}$. All data shown were expressed as mean $\pm \mathrm{s}$. $\mathrm{d}$. of three independent experiments. The images were from a representative experiment. ${ }^{\star} P<0.05,{ }^{\star} * P<0.01$.

associated with an increased risk of GC and has critical roles in tumour metastasis (Zhao et al, 2007). However, little is known about the exact effect of leptin on GC cell migration and its underlying mechanism. ICAM-1 is overexpressed in several cancers and implicated in tumour migration (Maruo et al, 2002; Chen et al, 2012). In this study, leptin and ICAM-1 levels were strongly correlated with each other in human GC tissues, as well as with clinical stage and lymph node metastasis, suggesting a causal connection between leptin pathway and ICAM-1. Our results also demonstrated that leptin may enhance the migration of GC cells (AGS and MKN-45) by upregulating ICAM-1 expression in vitro. Moreover, leptin induces the activation of Rho/ROCK pathway, which contributes to the subsequent upregulation of both surface ICAM-1 and sICAM-1. Taken together, our findings indicate that leptin enhances GC migration by increasing ICAM-1 expression through Rho/ROCK pathway.

Metastasis is a multi-step process during which cancer cells detach from the primary tumour, migrate, adhere, and invade into the blood or lymphatic vessels. Finally cancer cells extravasate out of the vessel and interact with the target tissue. Although multiple factors are involved in this process, adipocyte provides fatty acids for rapid tumour growth, and the dysfunction of lipid metabolism can lead to the pathogenesis of GC (Song et al, 2012). Leptin, secreted predominantly from adipocytes, may promote GC cell proliferation, suggesting an essential role of leptin in the progression of GC (Pai et al, 2005; Shida et al, 2005). This study has indicated that leptin induces the migration of GC cells (AGS and MKN-45) in a dose- and time-dependent manner, which keeps in line with previous studies that leptin induces migration of endometrial cancer and glioma cells (Yeh et al, 2009; $\mathrm{Wu}$ et al, 2012). Further clarifying the mechanisms responsible for leptin-induced GC metastasis is important for better understanding and control of tumour metastasis in patients with GC.

Our results revealed that leptin and ICAM-1 were overexpressed in GC tissue and correlated with lymph node metastasis, which is consistent with the previous studies (Maruo et al, 2002; Zhao et al, 2007). Further analysis indicated that leptin was positively correlated with ICAM-1, suggesting a possible link between leptin and ICAM-1 in the progression of GC. This is also supported by the findings that leptin and ICAM-1 are elevated after stimulation with inflammatory cytokines, for example, in patients with H. pylori infection (Fan et al, 1995; Azuma et al, 2001). Previous studies show that ICAM-1 undergoes cleavage at its extracellular stalk domain, and release a soluble form of the protein (sICAM-1), and this process may be mediated by MT1-MMP (MMP14) (Essick et al, 2008). In this study, we found that leptin enhanced the expression of ICAM-1, as well as cell surface ICAM-1 and sICAM-1 in GC cells. Furthermore, we also observed an enhanced expression of MT1-MMP by leptin in GC cells (Dong et al, 2013). Therefore, we speculate that leptin promotes ICAM-1 expression and subsequently enhances the level of sICAM-1 via upregulating MT1-MMP. Increased ICAM-1 may promote the adhesion of cancer cells, leading to tumour growth and mediating cellextracellular matrix interaction. When metastasis occurs, cancer cells detach from the primary lesion, penetrate through the tissue basement membrane, and invade blood vessels and lymphatic vessels. On the other hand, as the host killer cells (NK cells, LAK cells, and CTLs) and macrophages express LFA-1 and Mac-1, two major receptors of ICAM-1, partially via which they recognise and attack the tumour cells (Diamond et al, 1990), upregulated sICAM-1 may inhibit NK and macrophage activity by competitively binding to LFA-1 and Mac-1 (Koyama, 1994). Thus, it is 

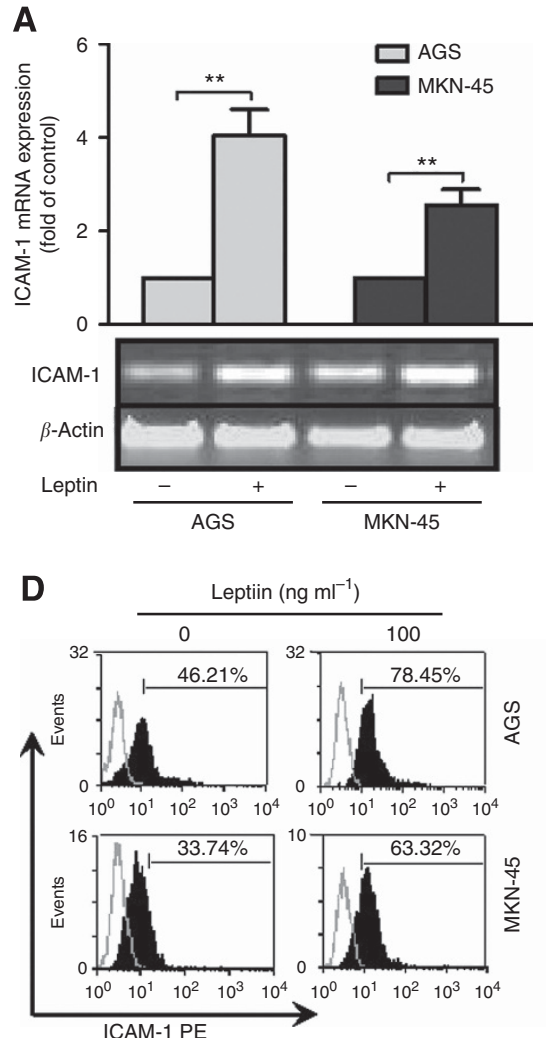

B
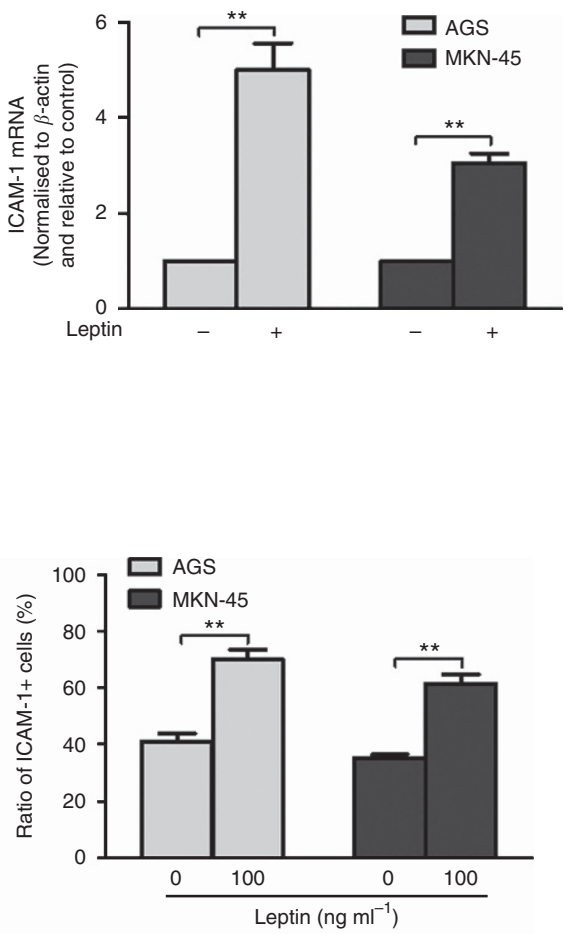

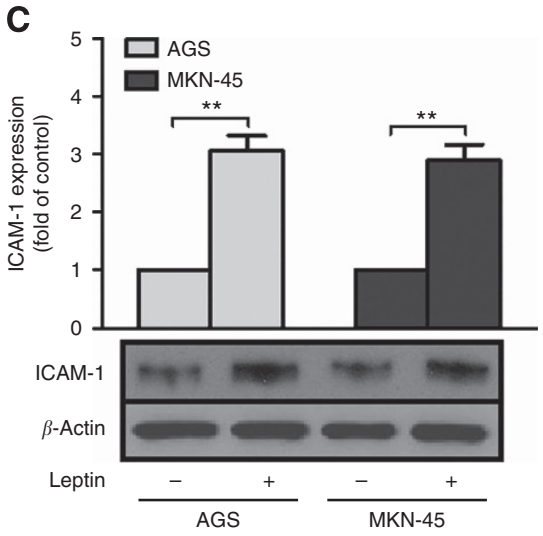

E

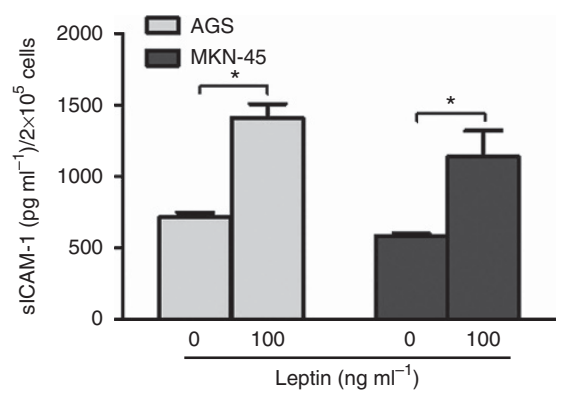

Figure 3. Leptin enhanced the expression of ICAM-1 in GC cells. AGS and MKN-45 cells were treated with leptin $\left(100 \mathrm{ng} \mathrm{ml}{ }^{-1}\right)$ for $24 \mathrm{~h}$. The expression of ICAM-1 was measured by RT-PCR (A), real time RT-PCR (B) and WB (C). The cell surface expression of ICAM-1 was measured by flow cytometry (D), and the level of sICAM-1 in supernatant was detected by ELISA (E). All data shown are expressed as mean \pm s.d. of three independent experiments. ${ }^{\star} P<0.05,{ }^{* \star} P<0.01$.
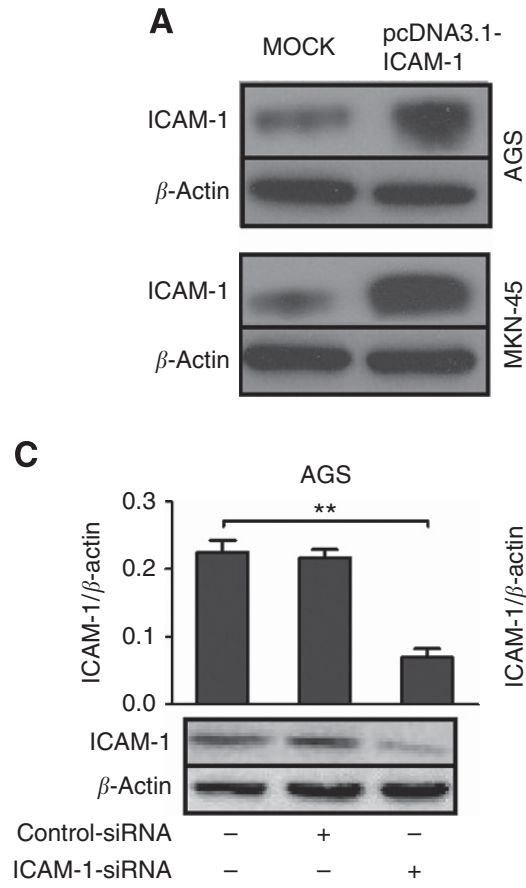

B

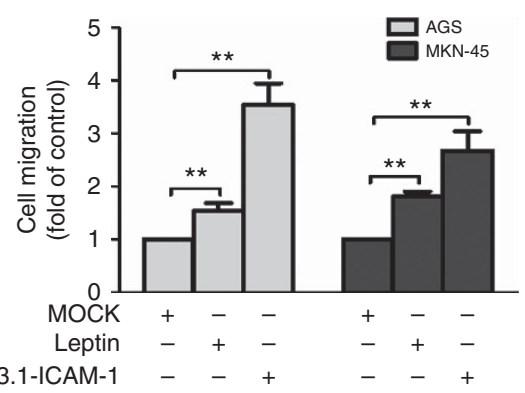

D

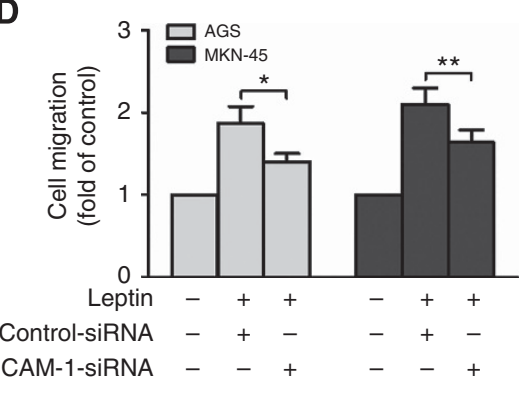

Figure 4. Leptin enhanced GC cell migration via upregulating ICAM-1. The expression level of ICAM-1 in GC cells was measured by WB after transfection with pCDNA3.1-ICAM-1 (A) or ICAM-1-siRNA (B). One representative result out of three independent experiments is shown. Graphs represent quantitation with $\beta$-actin as the internal standard. Each bar represents the mean $\pm \mathrm{s} . \mathrm{d}$. $(n=3, \star \star P<0.01)$. In addition, AGS and MKN-45 cells were transfected with pcDNA3.1-ICAM-1 (C) or ICAM-1-siRNA (D) for $24 \mathrm{~h}$ and then treated with leptin (100 $\mathrm{ng} \mathrm{ml-1}$ ) for $24 \mathrm{~h}$. The migration was measured by transwell assay. The migration numbers were expressed as relative to control. ${ }^{\star} P<0.05,{ }^{\star \star} P<0.01$. 


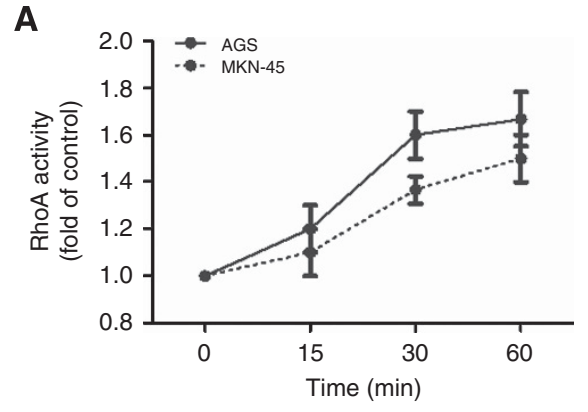

C
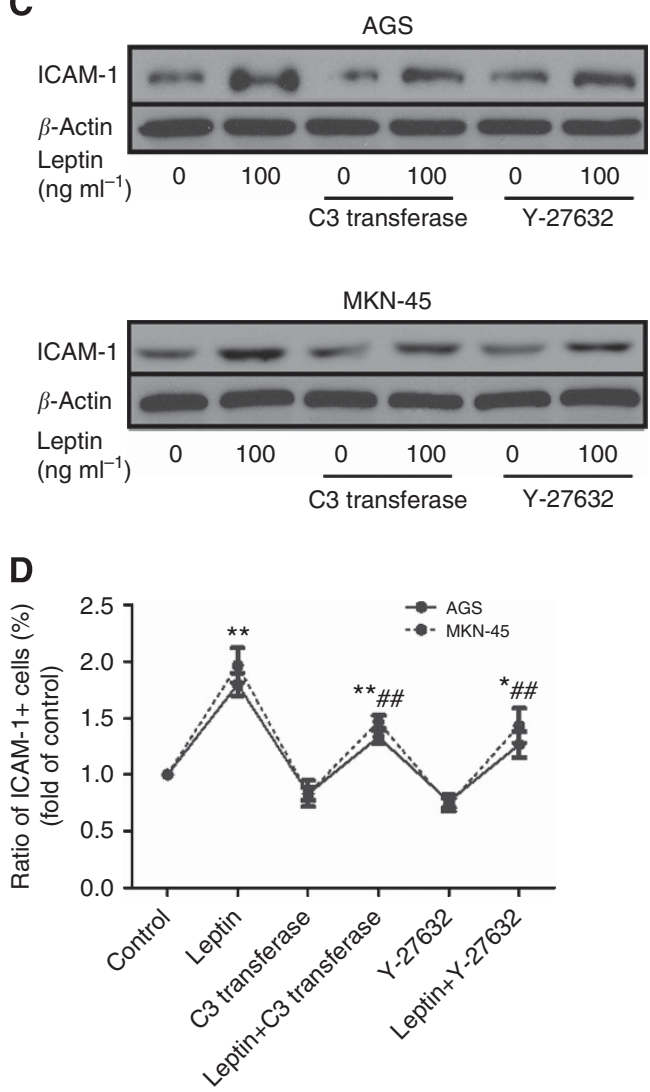

B
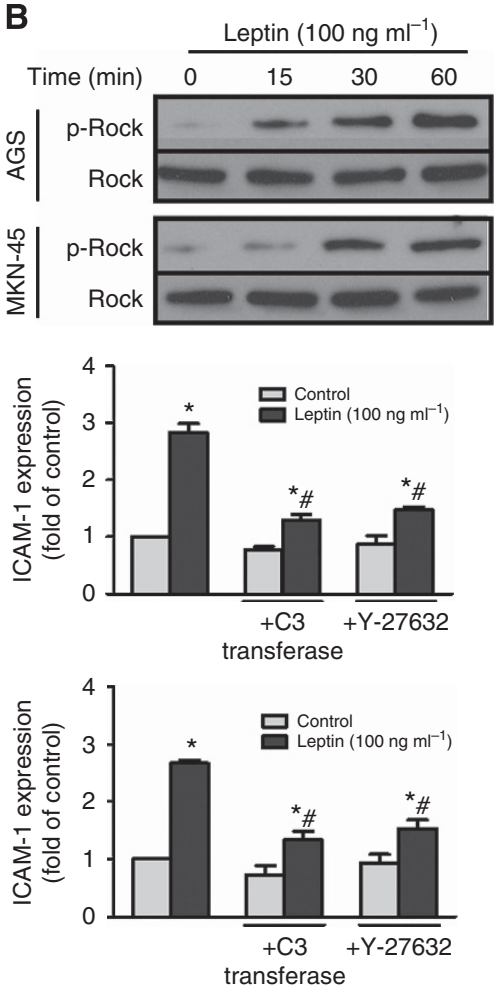

$\mathbf{E}$

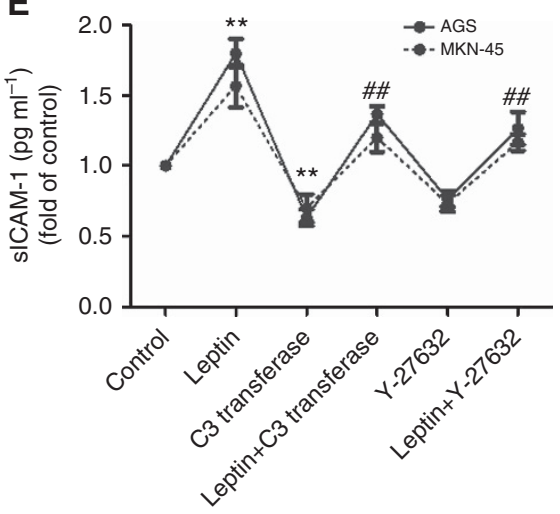

Figure 5. Rho/ROCK pathway was involved in the leptin-induced ICAM-1 expression in GC cells. AGS and MKN-45 cells were treated with leptin $\left(100 \mathrm{ng} \mathrm{ml}^{-1}\right)$ for various times $(0,15,30$, and $60 \mathrm{~min})$. The RhoA activity was detected by ELISA (A), and the phosphorylation levels of ROCK in cell lysates were determined by WB (B). In a separate experiment, AGS and MKN-45 cells were treated in the absence (control) or presence of leptin $\left(100 \mathrm{ng} \mathrm{ml}^{-1}\right)$ after $1 \mathrm{~h}$ pretreatment with $\mathrm{C} 3$ transferase $\left(0.25 \mu \mathrm{g} \mathrm{ml}^{-1}\right)$ or $\mathrm{Y}-27632(3.3 \mu \mathrm{M})$. The inhibitors were present for the duration of leptin treatment. After $24 \mathrm{~h}$, the levels of ICAM-1 (C), cell surface ICAM-1 (D), and sICAM-1 (E) in the presence or absence of C3 transferase or Y-27632 were determined. One representative result out of three independent experiments is shown. $\beta$-actin was used as an internal control. The results are shown as mean \pm s.d. ${ }^{*} P<0.05$ vs control, ${ }^{*} P<0.01$ vs control, ${ }^{\#} P<0.05$ vs control, ${ }^{\# \#} P<0.01$ vs the group of leptin.

possible that there is a balance between ICAM-1/sICAM-1 and immunocytes, which can also be explained by the fact that it takes a long time for detectable tumour to form a metastatic lesion. With the development of GC, leptin increased sICAM-1 level, breaking the balance and subsequently resulting in a failure of the host immunocytes to effectively kill the tumour cells, which may have, therefore, protected the tumour cells from elimination and facilitated tumour migration. The hypothesis is well supported by previous findings that serum leptin and SICAM-1 are elevated and positively correlated with the clinical stage and lymph node metastasis of tumour (Maruo et al, 2002; Capelle et al, 2009). Taken together, these results suggest that leptin/ICAM-1/sICAM-1 is implicated in GC metastasis and may thus provide a potential therapeutic target against GC, which needs to be further confirmed by more experiments with animal models.
Sunami et al (2000) have shown that lymph node metastasis reduced after successful transfection of ICAM-1 gene into the cancer cells. Because of the enhancement of immune surveillance mechanism, tumour cells might be easily captured and destroyed by the lymphocytes. However, their cell model is different from ours, in addition, these authors did not discuss the production of sICAM-1 in their ICAM-1-overexpressing cells, though previous study from the same group revealed that the survival rate in high sICAM-1 group significantly decreased compared with that in low sICAM-1 group (Nakata et al, 2000).

The present study has demonstrated the importance of leptininduced upregulation of ICAM-1 in GC cell migration and further elucidated the underlying mechanism. It is well known that the activity of small GTPases (including RhoA) is important for the signalling derived from lipid metabolism (Bolick et al, 2005). 


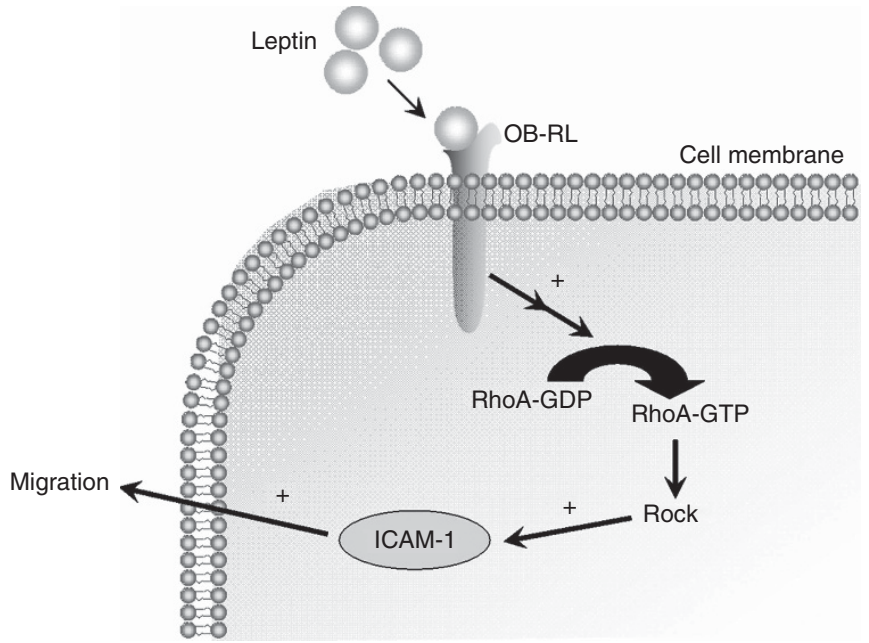

Figure 6. Schematic diagram summarising the leptin-RhoA/ROCKICAM-1 pathway on the migration of GC cells. By binding with OB-RL, leptin activates the RhoA/ROCK pathway, which subsequently induces the expression of ICAM-1. All of these finally promoted migration of GC. Abbreviation: OB-RL= leptin receptor.

This study has shown that leptin may activate Rho/ROCK pathway, which is consistent with the previous findings that leptin regulates myocardial matrix remodelling by activating Rho/ROCK (Schram et al, 2011). Further analysis indicates that the inhibitor of either Rho (C3 transferase) or ROCK (Y27632) attenuates leptin-induced ICAM-1 expression, illustrating that the use of ROCK inhibitors might be a potential therapeutic approach. This is also supported by the finding that Rho/ROCK pathway critically regulates the plasticity of metastatic GC (Matsuoka et al, 2011). Notably, the inhibitor treatment in this study failed to completely block the leptin-induced upregulation of ICAM-1, implying a possible contribution from other unidentified leptin-activated pathway(s). Several signalling pathways activated by leptin have been identified in GC, that is, Janus kinase/signal transducers and activators of transcription (JAK/STAT), extracellular signal-regulated kinase 1/2 (ERK1/2), and mitogen-activated protein kinase (MAPK) pathways, as well as phosphatidylinositol 3-kinase (PI3K)/AKT pathways (Pai et al, 2005; Shida et al, 2005). Previous study reveals that leptin upregulates ICAM-1 expression in eosinophils by the combined activation of MAPK and JAK pathways under allergic inflammation (Wong et al, 2007). However, their changes were not examined in this study. Although we cannot exclude the possible effect of these signalling pathways, the upregulation of ICAM-1 induced by leptin in the cells of this study might primarily depend on the RhoA/ROCK pathway. Further experiments will be conducted to test these possibilities. In addition, Rho GTPases are the binary switch between an active GTP-bound form and an inactive GDP-bound form. This process might be mediated by several molecules, such as Rho guanine nucleotide exchange factors (Rossman et al, 2005), NF- $\kappa \mathrm{B}$ (Jung et al, 2012), and reactive oxygen species (Jin et al, 2004). Although these mechanisms remain an interesting research topic, they are beyond the scope of the current study.

In conclusion, this study has shown that leptin enhances GC cell migration by increasing ICAM-1 expression, which is demonstrated to be largely dependent on the activation of Rho/ROCK signalling pathway. A summarised schematic diagram is shown in Figure 6. Our results may provide preliminary experimental clues for future development of novel therapies against the metastasis of GC.

\section{ACKNOWLEDGEMENTS}

The project was supported by the China National Natural Science Foundation Projects (Grant No. 81072406, 81271916, 31270971, and 81301506), Research Fund for the Doctoral Program of Higher Education of China (Grant No. 20120131110055), Shandong Province Natural Science Foundation (Grant No. ZR2010HZ004). The authors thank Professor Jian-Jun Wei (Department of Pathology, Robert H. Lurie Comprehensive Cancer Center of Northwestern University) for advice on experimental design and critically reviewing the manuscript.

CONFLICT OF INTEREST

The authors declare no conflict of interest.

\section{REFERENCES}

Azuma T, Suto H, Ito Y, Ohtani M, Dojo M, Kuriyama M, Kato T (2001) Gastric leptin and Helicobacter pylori infection. Gut 49: 324-329.

Bolick DT, Orr AW, Whetzel A, Srinivasan S, Hatley ME, Schwartz MA, Hedrick CC (2005) 12/15-lipoxygenase regulates intercellular adhesion molecule-1 expression and monocyte adhesion to endothelium through activation of RhoA and nuclear factor-kappaB. Arterioscler Thromb Vasc Biol 25: 2301-2307.

Burridge K, Wennerberg K (2004) Rho and Rac take center stage. Cell 116: 167-179.

Calle EE, Rodriguez C, Walker-Thurmond K, Thun MJ (2003) Overweight, obesity, and mortality from cancer in a prospectively studied cohort of U.S. adults. N Engl J Med 348: 1625-1638.

Capelle LG, de Vries AC, Haringsma J, Steyerberg EW, Looman CW, Nagtzaam NM, van Dekken H, ter Borg F, de Vries RA, Kuipers EJ (2009) Serum levels of leptin as marker for patients at high risk of gastric cancer. Helicobacter 14: 596-604.

Chan JL, Matarese G, Shetty GK, Raciti P, Kelesidis I, Aufiero D, De Rosa V, Perna F, Fontana S, Mantzoros CS (2006) Differential regulation of metabolic, neuroendocrine, and immune function by leptin in humans. Proc Natl Acad Sci USA 103: 8481-8486.

Chen PC, Lin TH, Cheng HC, Tang CH (2012) CCN3 increases cell motility and ICAM-1 expression in prostate cancer cells. Carcinogenesis 33: 937-945.

Diamond MS, Staunton DE, de Fougerolles AR, Stacker SA, Garcia-Aguilar J, Hibbs ML, Springer TA (1990) ICAM-1 (CD54): a counter-receptor for Mac-1 (CD11b/CD18). J Cell Biol 111: 3129-3139.

Dong Z, Xu X, Du L, Yang Y, Cheng H, Zhang X, Li Z, Wang L, Li J, Liu H, Qu X, Wang C (2013) Leptin-mediated regulation of MT1-MMP localization is KIF1B dependent and enhances gastric cancer cell invasion. Carcinogenesis 34: 974-983.

Essick E, Sithu S, Dean W, D'Souza S (2008) Pervanadate-induced shedding of the intercellular adhesion molecule (ICAM)-1 ectodomain is mediated by membrane type-1 matrix metalloproteinase (MT1-MMP). Mol Cell Biochem 314: 151-159.

Fan XG, Fan XJ, Xia HX, Keeling PW, Kelleher D (1995) Up-regulation of CD44 and ICAM-1 expression on gastric epithelial cells by $\mathrm{H}$. pylori. Apmis 103: 744-748.

Grothey A, Heistermann P, Philippou S, Voigtmann R (1998) Serum levels of soluble intercellular adhesion molecule-1 (ICAM-1, CD54) in patients with non-small-cell lung cancer: correlation with histological expression of ICAM-1 and tumour stage. Br J Cancer 77 : 801-807.

Howard JM, Pidgeon GP, Reynolds JV (2010) Leptin and gastro-intestinal malignancies. Obes Rev 11: 863-874.

Ishikawa M, Kitayama J, Nagawa H (2006) Expression pattern of leptin and leptin receptor (OB-R) in human gastric cancer. World J Gastroenterol 12: $5517-5522$.

Jin L, Ying Z, Webb RC (2004) Activation of Rho/Rho kinase signaling pathway by reactive oxygen species in rat aorta. Am J Physiol Heart Circ Physiol 287: H1495-H1500. 
Jung CH, Lee WJ, Hwang JY, Seol SM, Kim YM, Lee YL, Ahn JH, Park JY (2012) The role of Rho/Rho-kinase pathway in the expression of ICAM-1 by linoleic acid in human aortic endothelial cells. Inflammation 35: 1041-1048.

Kaliora AC, Kountouri AM, Karathanos VT, Koumbi L, Papadopoulos NG, Andrikopoulos NK (2008) Effect of Greek raisins (Vitis vinifera L.) from different origins on gastric cancer cell growth. Nutr Cancer 60: 792-799.

Koyama S (1994) Immunosuppressive effect of shedding intercellular adhesion molecule 1 antigen on cell-mediated cytotoxicity against tumor cells. Jpn J Cancer Res 85: 131-134.

Makrilia N, Kollias A, Manolopoulos L, Syrigos K (2009) Cell adhesion molecules: role and clinical significance in cancer. Cancer Invest 27: 1023-1037.

Maruo Y, Gochi A, Kaihara A, Shimamura H, Yamada T, Tanaka N, Orita K (2002) ICAM-1 expression and the soluble ICAM-1 level for evaluating the metastatic potential of gastric cancer. Int J Cancer 100: 486-490.

Matsuoka T, Yashiro M, Kato Y, Shinto O, Kashiwagi S, Hirakawa K (2011) RhoA/ROCK signaling mediates plasticity of scirrhous gastric carcinoma motility. Clin Exp Metastasis 28: 627-636.

Nakata B, Hori T, Sunami T, Ogawa Y, Yashiro M, Maeda K, Sawada T, Kato Y, Ishikawa T, Hirakawa K (2000) Clinical significance of serum soluble intercellular adhesion molecule 1 in gastric cancer. Clin Cancer Res 6: 1175-1179.

Otvos Jr L, Kovalszky I, Riolfi M, Ferla R, Olah J, Sztodola A, Nama K, Molino A, Piubello Q, Wade JD, Surmacz E (2011) Efficacy of a leptin receptor antagonist peptide in a mouse model of triple-negative breast cancer. Eur J Cancer 47: 1578-1584.

Pai R, Lin C, Tran T, Tarnawski A (2005) Leptin activates STAT and ERK2 pathways and induces gastric cancer cell proliferation. Biochem Biophys Res Commun 331: 984-992.

Parkin DM, Bray F, Ferlay J, Pisani P (2005) Global cancer statistics, 2002. CA Cancer J Clin 55: 74-108.

Polychronidis AC, Tsaroucha AK, Samolis SP, Botaitis SK, Perente SS, Simopoulos CE (2003) Serum levels of intercellular adhesion molecule-1 correlate with advanced and metastatic disease and poor prognosis in gastric cancer. Folia Med (Plovdiv) 45: 20-24.

Rossman KL, Der CJ, Sondek J (2005) GEF means go: turning on RHO GTPases with guanine nucleotide-exchange factors. Nat Rev Mol Cell Biol 6: $167-180$.

Sahai E, Marshall CJ (2002) RHO-GTPases and cancer. Nat Rev Cancer 2: 133-142.

Sawada K, Morishige K, Tahara M, Ikebuchi Y, Kawagishi R, Tasaka K, Murata Y (2002) Lysophosphatidic acid induces focal adhesion assembly through Rho/Rho-associated kinase pathway in human ovarian cancer cells. Gynecol Oncol 87: 252-259.

Schram K, Ganguly R, No EK, Fang X, Thong FS, Sweeney G (2011) Regulation of MT1-MMP and MMP-2 by leptin in cardiac fibroblasts involves Rho/ROCK-dependent actin cytoskeletal reorganization and leads to enhanced cell migration. Endocrinology 152: 2037-2047.

Shida D, Kitayama J, Mori K, Watanabe T, Nagawa H (2005) Transactivation of epidermal growth factor receptor is involved in leptin-induced activation of janus-activated kinase 2 and extracellular signal-regulated kinase 1/2 in human gastric cancer cells. Cancer Res 65: 9159-9163.

Song H, Peng JS, Yao DS, Liu DL, Yang ZL, Du YP, Xiang J (2012) Metabolic disorders of fatty acids and fatty acid amides associated with human gastric cancer morbidity. Chin Med J (Engl) 125: 757-763.

Sunami T, Yashiro M, Chung KH (2000) ICAM-1 (intercellular adhesion molecule-1) gene transfection inhibits lymph node metastasis by human gastric cancer cells. Jpn J Cancer Res 91: 925-933.

Wang Z, Chen JQ (2011) Imaging in assessing hepatic and peritoneal metastases of gastric cancer: a systematic review. BMC Gastroenterol 11: 19.

Wong CK, Cheung PF, Lam CW (2007) Leptin-mediated cytokine release and migration of eosinophils: implications for immunopathophysiology of allergic inflammation. Eur J Immunol 37: 2337-2348.

Wu X, Yan Q, Zhang Z, Du G, Wan X (2012) Acrp30 inhibits leptin-induced metastasis by downregulating the JAK/STAT3 pathway via AMPK activation in aggressive SPEC-2 endometrial cancer cells. Oncol Rep 27: $1488-1496$.

Xu X, Dong Z, Li Y, Yang Y, Yuan Z, Qu X, Kong B (2013) The upregulation of signal transducer and activator of transcription 5-dependent microRNA-182 and microRNA-96 promotes ovarian cancer cell proliferation by targeting forkhead box $\mathrm{O} 3$ upon leptin stimulation. Int J Biochem Cell Biol 45: 536-545.

Xu X, Wang Q, Deng B, Wang H, Dong Z, Qu X, Kong B (2012) Monocyte chemoattractant protein-1 secreted by decidual stromal cells inhibits NK cells cytotoxicity by up-regulating expression of SOCS3. PLoS One 7: e41869.

Yang YM, Feng AL, Zhou CJ, Liang XH, Mao HT, Deng BP, Yan S, Sun JT, Du LT, Liu J, Wang QJ, Neckenig MR, Yang QF, Qu X (2011) Aberrant expression of chemokine receptor CCR4 in human gastric cancer contributes to tumor-induced immunosuppression. Cancer Sci 102: 1264-1271.

Yeh WL, Lu DY, Lee MJ, Fu WM (2009) Leptin induces migration and invasion of glioma cells through MMP-13 production. Glia 57: 454-464.

Yu JA, Sadaria MR, Meng X, Mitra S, Ao L, Fullerton DA, Weyant MJ (2012) Lung cancer cell invasion and expression of intercellular adhesion molecule-1 (ICAM-1) are attenuated by secretory phospholipase A(2) inhibition. J Thorac Cardiovasc Surg 143: 405-411.

Zhao X, Huang K, Zhu Z, Chen S, Hu R (2007) Correlation between expression of leptin and clinicopathological features and prognosis in patients with gastric cancer. J Gastroenterol Hepatol 22: 1317-1321.

Zimmerman T, Blanco FJ (2008) Inhibitors targeting the LFA-1/ICAM-1 cell-adhesion interaction: design and mechanism of action. Curr Pharm Des 14: $2128-2139$.

This work is published under the standard license to publish agreement. After 12 months the work will become freely available and the license terms will switch to a Creative Commons AttributionNonCommercial-Share Alike 3.0 Unported License. 